\title{
Régulation métabolique de l'ingestion chez les monogastriques *
}

\author{
J Louis-Sylvestre \\ Université $P$ et $M$ Curie, Laboratoire de neurobiologie de la nutrition, \\ 4, Place Jussieu, 75252 Paris cedex 05, France
}

(Reçu le 24 septembre 1990; accepté le 13 février 1991)

\begin{abstract}
Résumé - Chez le sujet animal ou humain, le maintien des réserves énergétiques à un niveau constant implique que les dépenses et/ou les entrées d'énergie soient rétrorégulées par ce niveau. La revue des connaissances actuelles concernant, d'une part, le signal interne qui déclenche l'acte alimentaire et, d'autre part, le mécanisme lipostatique, permet de faire l'hypothèse selon laquelle le mécanisme lipostatique pourrait affecter la prise alimentaire par l'intermédiaire de son signal de déclenchement. II semble, en effet, que l'activité des neurones du centre hypothalamique ventromédian (HVM) reflète l'état métabolique périphérique et l'état des réserves. Cette activité commanderait de façon adaptée, soit le retrait des métabolites circulants vers les réserves, soit leur libération à partir des mêmes réserves. Ces mouvements précipiteraient ou retarderaient la survenue d'un signal glucopénique initiateur du repas.
\end{abstract}

prise alimentaire / hypoglycémie préprandiale / mécanisme lipostatique / insuline / hypothalamus

Summary - Metabolic regulation of ingestion in monogastric animals. Evidence has accumulated over the past 2 decades to show that the regulation of the fat body mass is integrated in the control of food intake. It was suggested long ago that the metabolic stimulation to eat originated from a shortage of available circulating metabolites; particularly, glucose uptake and utilization has been a central feature of many hypotheses since Mayer's glucostatic theory. In the first section of this contribution, evidence is presented that in rats and man a premeal decline in blood glucose concentration is causally related to meal initiation. The origin of the decline and the possible role of "glycemiasensitive" neurons located in the lateral hypothalamus $(\mathrm{LH})$ as central sensors of this metabolic stimulus to eat are discussed. In the second section, it is suggested that LH energy utilization and ventro-medial hypothalamus (VMH) energy storage reflect metabolic change at the periphery and also determined the neuronal activity which monitors storage or mobilization of fuels into and from stores. Since glucose is a precursor of triglycerides deposited in adipose tissue and since oxidation of fatty acids in peripheral tissues has a glucose-sparing effect, circulating glucose seems a likely candidate for the signal mediating the effect of fat deposits on food intake.

\section{food intake / preprandial hypoglycemia / lipostatic mechanism / insulln / hypothalamus}

\footnotetext{
* Ce texte correspond à l'essentiel d'une communication faite le 11 septembre 1990 à Rennes, à l'occasion du colloque "Ingestion-Digestion-Absorption et Science des Aliments» organisé par l'Association française de nutrition.
} 


\section{INTRODUCTION}

Le comportement alimentaire est la première étape d'un processus qui permet que soient couverts les besoins variables mais continus de l'organisme en énergie et éléments spécifiques. Dans la majorité des espèces, le comportement alimentaire est discontinu et l'approvisionnement continu des cellules n'est possible que parce qu'existent des réserves.

L'acte alimentaire est le remplissage d'un réservoir de petite capacité, le tractus gastro-intestinal, dont la $2^{e}$ fonction est la transformation des aliments en métabolites. Ceux-ci entrent dans la circulation qui les distribue aux tissus, soit pour usage immédiat, soit pour stockage de plus ou moins longue durée dans les tissus spécialisés, principalement le tissu adipeux. Dans cet organe spécialisé, les réserves condensées permettent le repos et la survie en cas de disette; elles ne sont efficaces que parce que leur niveau est, dans certaines limites, maintenu constant.

Le maintien des réserves à un niveau constant implique que les dépenses etou la prise alimentaire soient rétrorégulées directement ou indirectement par ce niveau. Les possibilités d'adaptation des dépenses d'un organisme étant très limitées, l'effecteur principal de la régulation est évidemment la prise alimentaire. La revue des connaissances actuelles concernant, d'une part le signal interne qui déclenche l'acte alimentaire, et d'autre part le mécanisme lipostatique, va permettre de montrer comment ce mécanisme lipostatique affecte la prise alimentaire, et ce, par l'intermédiaire de son signal de déclenchement.

\section{LE STIMULUS INTERNE DÉCLEN- CHANT LA PRISE ALIMENTAIRE}

Quel signal déclenche l'acte alimentaire chez le sujet en conditions stables ayant continuellement des aliments à disposition?

Au XVIII siècle, les premiers physiologistes qui se sont penchés sur cette question pensaient que les contractions de l'estomac vide pouvaient être à l'origine de la sensation de faim et de la prise d'un repas. Depuis, les hypothèses se sont succédé et ont évoqué, tour à tour, des variations osmotiques du milieu intérieur, une baisse de température interne, une augmentation du taux des acides gras libres, un changement du spectre des acides aminés circulants. Cependant, aucune de ces hypothèses ne tient à l'examen (voir revue Le Magnen, 1984).

En 1974, Nicolaïdis présentait l'hypothèse "ischymétrique" et proposait que le signal de faim soit une baisse de l'utilisation par les cellules de l'ensemble des métabolites énergétiques (glucides, lipides, protides). Even et Nicolaïdis (1984) montraient que tout repas est précédé d'une chute du métabolisme de fond, ensemble des dépenses énergétiques de l'organisme à l'exception de celle due à l'activité motrice. Cependant, si l'injection de 2désoxyglucose (2DG) qui bloque l'utilisation cellulaire des glucides provoque bien une baisse du métabolisme de fond et une hyperphagie, l'injection d'acide nicotinique qui bloque l'utilisation des lipides et provoque une baisse du métabolisme de fond n'affecte pas la prise alimentaire (Even et al, 1988). Par ailleurs, cette théorie ne précise pas l'origine de la baisse incriminée. 
En 1953, Van Itallie et al montraient que, chez le sujet humain, la sensation de faim apparaît quand la différence artérioveineuse de glucose s'annule. Mayer (1955) proposait alors la théorie dite glucostatique selon laquelle le stimulus recherché serait une baisse de l'approvisionnement cellulaire en glucose; il précisait bien que le taux de glucose en soi ne pouvait être le facteur déterminant puisque peuvent coexister état de faim et hyperglycémie. Cette théorie a suscité un nombre considérable de travaux (voir revue Le Magnen, 1984) qui relèvent des 2 techniques d'exploration possibles. L'une consiste à provoquer par diverses manipulations expérimentales des changements du milieu intérieur et à observer le comportement alimentaire induit. L'autre est la recherche d'une corrélation temporelle et quantitative entre des événements métaboliques ou neuro-endocriniens et les événements alimentaires.

La première voie a conduit à la conclusion qu'une glucopénie cellulaire artificiellement induite stimule la prise alimentaire; la question de savoir si cette action reproduit celle qui physiologiquement active le comportement ingestif reste posée. La deuxième approche, qui est l'étude simultanée de divers paramètres sanguins et de la prise alimentaire spontanée chez l'animal libre de ses mouvements, non perturbé par les prélèvements, fut employée par l'équipe de Steffens (Steffens 1969, 1970; Strubbe et Steffens, 1977; Strubbe et al, 1977; Strubbe et Bouman, 1978). Par des prélèvements sanguins effectués toutes les 10 min sur le rat disposant de nourriture à volonté, ces chercheurs montraient que la glycémie est parfaitement stable entre les repas, que l'insulinémie décline progressivement et atteint le niveau le plus bas quand commence le repas. Le taux sanguin des acides gras, bas à cet instant, s'élève quand régresse l'hyperglycémie postabsorptive. Quand le repas est empêché, l'euglycémie n'est maintenue que pendant une vingtaine de min; une chute lente est observée ensuite. Ces auteurs concluaient alors qu'aucun de ces paramètres, en l'absence de variations systématiques avant le repas, ne peut être le signal responsable de l'acte alimentaire.

Dans ce travail, les prélèvements sanguins étant effectués toutes les $10 \mathrm{~min}$, et les valeurs rapportées étant des moyennes, la dernière valeur de glycémie "préprandiale" représente la glycémie de l'animal environ 5 min avant le repas. Une technique de prélèvement sanguin et dosage de la glycémie en continu pendant 5 h (les $15 \mu \mathrm{l} / \mathrm{min}$ de sang prélevé sont remplacés par un volume égal de sang prélevé sur un congénère et perfusé par voie fémorale) a été utilisée chez le rat libre de ses mouvements, disposant à volonté de sa nourriture habituelle (Louis-Sylvestre et Le Magnen, 1980a). Elle a permis de montrer que tous les repas spontanés sont précédés d'une chute de la glycémie. Cette baisse progressive commence 5-6 min avant le début de l'ingestion et se poursuit pendant environ 3 min après; elle est suivie de l'hyperglycémie postingestive. Entre les repas, la glycémie est stable; les variations aléatoires toujours brèves, ne dépassent pas $6 \%$. Une réplique des précédents travaux réalisée par Campfield et al (1985) a confirmé l'existence d'une hypoglycémie préprandiale.

Ainsi, une corrélation temporelle était établie entre un événement métabolique et un événement alimentaire : chez le rat nourri ad libitum, non perturbé, tout repas spontané suit une hypoglycémie et toute hypoglycémie de ce type précède un repas. Un rapport de causalité pouvait-il être établi ? Le Magnen a montré que chez le rat en conditions stables, disposant d'un aliment complet équilibré, il existe une correlation hautement significative entre la di- 
mension calorique du repas et l'intervalle postprandial (Le Magnen et Devos, 1980). L'existence de cette relation est d'ailleurs un argument en faveur du déclenchement de l'ingestion par le manque de carburant immédiatement disponible. Connaissant l'heure et la dimension d'un certain repas, on peut prévoir l'heure du repas suivant. II a été alors possible de montrer qu'en absence d'aliments, le phénomène préprandial a lieu. Cette hypoglycémie est alors rapidement, mais momentanément corrigée (20 min environ), puis une seconde hypoglycémie, moins bien corrigée que la première, apparaît. De la même façon, il a été montré que toute intervention qui prévient la chute préprandiale de la glycémie retarde le repas. Celui-ci n'intervient que lorsque la glycémie baisse à nouveau. Ainsi chez le rat, une injection intraveineuse d'adrénaline $(0,96 \mathrm{nmol} / 100 \mathrm{~g})$ faite $15 \mathrm{~min}$ avant l'heure présumée du repas crée une hyperglycémie d'environ $7 \%$. Environ 60 min plus tard intervient une hypoglycémie suivie du repas dont la taille n'est pas modifiée. Un stress nociceptif (queue dans l'eau à $51^{\circ} \mathrm{C}$ ) imposé à l'animal lui aussi $15 \mathrm{~min}$ avant l'heure présumée, induit une hyperglycémie de $6 \%$; le phénomène hypoglycémique qui intervient avec délai est suivi du repas qui accuse un retard d'environ 27 min (Lagaillarde, 1985). Campfield et al (1984) corrigent l'hypoglycémie précocément détectée par une injection ( $5 \mathrm{mg}$ ) puis une perfusion (3 $\mathrm{mg} /$ $\mathrm{min}$ ) de glucose; le repas est alors retardé de 8 à à $600 \mathrm{~min}$. Lorsque l'hypoglycémie est corrigée utrop tard" (1 cas) le repas survient malgré l'intervention. À la lumière de ces travaux, une relation de causalité peut être évoquée : I'hypoglycémie étant transitoirement empêchée, le repas est retardé et ce, proportionnellement à la durée de la correction.

La cascade d'événements qui explique que les 2 interventions, injection d'adréna- line ou stress soient hyperglycémiantes est bien établie. L'adrénaline inhibe la sécrétion d'insuline, active celle du glucagon, réduit le retrait du glucose par le foie et les tissus périphériques, réduit indirectement la glycogénèse au niveau hépatique et musculaire; elle stimule le relargage des acides gras par le tissu adipeux, la synthèse des acides aminés participant à la gluconéogenèse, la glycogénolyse hépatique et musculaire libérant ainsi des métabolites participant à la gluconéogenèse (lactate en particulier).

Un stress aigu, tel que celui qui fut employé, induit une activation sympathique et une inhibition parasympathique. L'effet des efférences nerveuses directes sur le foie, le pancréas, le tissu adipeux, s'ajoute à la stimulation médullosurénalienne. Le stress provoque aussi une augmentation rapide d'ACTH et donc de corticostérone qui participe à la stimulation de la glycogénolyse.

Les 2 interventions, l'injection d'adrénaline et le stress ponctuel, retardent-elles le repas parce que l'afflux de glucose qu'elles provoquent prévient momentanément la glucopénie cellulaire ou parce qu'elles sont intrinsèquement anorexigènes ?

Pour tenter de répondre, l'effet sur un repas conditionné des stimuli hyperglycémiants précédemment décrits a été recherché. Ce conditionnement alimentaire a été bien étudié par Weingarten (1983) chez le rat. Pendant une dizaine de jours le rat est nourri à heures fixes et chacun des repas est précédé de 2 stimulus visuel et auditif. La nourriture étant ensuite continuellement disponible, l'animal reprend son alimentation spontanée mais fait un repas supplémentaire de taille normale chaque fois que surviennent les stimulus. L'animal compense ces repas supplémentaires en mangeant moins sur le reste des $24 \mathrm{~h}$ : la prise quotidienne totale n'est pas modifiée. Répondant à une stimulation externe, ces repas ingérés par un rat rassasié ne sont 
évidemment pas induits par une glucopénie cellulaire. Il a été vérifié qu'aucune des manœuvres hyperglycémiantes efficaces sur le repas spontané ne modifiait ni la latence, ni la dimension du repas conditionné (Lagaillarde, 1985).

\section{Quelle est l'origine du phénomène préprandial ?}

L'origine de l'hypoglycémie préprandiale est encore hypothétique. La réserve hépatique de glycogène du rat nourri ad libitum n'est jamais libérée en totalité par le foie; partiellement utilisée en période de repos, elle augmente de repas en repas pendant la période d'activité (Fuller et Diller, 1976; Le Magnen et Devos, 1980). Un épuisement du glycogène hépatique n'est donc pas impliqué.

L'hypoglycémie préprandiale pourrait être due à l'épuisement de la réserve gastro-intestinale (donc à un manque d'apport de métabolites au foie) dont de Castro (1981) a suggéré qu'il pouvait déterminer l'intervalle interprandial. À l'appui de cette hypothèse on peut remarquer que, quelle qu'en soit la cause, une accélération de l'absorption des nutriments est suivie d'une augmentation de la fréquence des repas (Puls et al, 1977; Glick et Modan, 1977; Thomas et Mayer, 1978; Geiselman et Novin, 1982).

Une troisième hypothèse est celle qui concerne une hypersécrétion transitoire d'insuline. Un tel pic a été mis en évidence avant ou au début de la baisse préprandiale de la glycémie et une corrélation temporelle a été observée entre le pic d'insuline, le début de l'hypoglycémie et le début du repas (Campfield et Smith, 1986). Le Magnen (1988) a suggéré que le pic d'insuline pourrait avoir pour origine une diminution de la concentration en glucose du contenu du tractus digestif à laquelle se- raient sensibles les glucorécepteurs intestinaux mis en évidence par Mei (1978). II est établi que certains sont susceptibles d'influencer l'insulinosécrétion (Mei et al, 1984).

\section{Quels récepteurs périphériques ou centraux sensibles à ce signal de faible amplitude peuvent intervenir dans la commande de l'acte alimentaire?}

Les glucorécepteurs intestinaux vagaux (Mei, 1978; Mei et al, 1981) et splanchniques (Perrin et al, 1981) étant activés par la présence de glucose dans l'intestin, il ne peut être exclu que leur inhibition intervienne dans le déclenchement de la prise alimentaire. Les glucorécepteurs hépatiques (Nijima, 1969) ont fait l'objet d'un nombre considérable de travaux qui démontrent leur rôle possible dans les mécanismes qui sous-tendent le comportement alimentaire; néanmoins, le fait que le comportement alimentaire (prise calorique, séquence) du rat après transplantation hépatique ne soit en rien modifié, démontre qu'ils ne sont indispensables ni pour le déclenchement, ni pour l'arrêt de l'acte alimentaire (Louis-Sylvestre et al, 1990).

Le rôle possible de glucorécepteurs ou de neurones glucosensibles centraux a été l'objet d'une revue exhaustive récente (Himmi, 1989). Seuls les points essentiels seront évoqués ici.

L'enregistrement in vitro de l'activité unitaire sur tranches d'encéphale a montré que les neurones des noyaux ventromédians (VMH), dorsomédians (DMH); ceux de l'aire latérale (LH) et du noyau du faisceau solitaire (NFS) et enfin ceux du bulbe sont affectés par la concentration en glucose dans le milieu. Par ailleurs, l'administration intracérébroventriculaire (ICV) d'antimétabolites du glucose, de phloridzine 
modifie la prise alimentaire : une glucoprivation centrale peut donc intervenir dans le comportement alimentaire.

Par applications locales, in vivo, de divers agents (glucose, aurothioglucose, etc), l'existence de glucorécepteurs ou de neurones glucosensibles a été démontrée au niveau du VMH, du LH, des noyaux paraventriculaires (PVN), du NFS et de l'area postrema (AP). En ce qui concerne le VMH, les expériences de lésion, stimulation, administration locales de diverses substances conduisent à penser que ces noyaux sont le site critique de régulation de la masse adipeuse et qu'ils jouent alors un rôle seulement indirect dans le comportement alimentaire (revue Le Magnen, 1984).

L'importance des structures rhombencéphaliques (NFS, AP) dans le déclenchement de la prise alimentaire en réponse à une glucoprivation a été montrée pour la première fois par Ritter et al (1981). Des neurones qui répondent à une application locale, à une perfusion de glucose dans la veine porte, ont été mis en évidence dans le NFS (Adachi et al, 1984); de plus, il existe des neurones glucosensibles dans I'AP (Adachi et Kobashi, 1985, 1988); or l'AP est un organe circumventriculaire non protégé par la barrière hématoencéphalique; ces neurones pourraient directement évaluer la concentration sanguine en glucose et intervenir dans la commande de prise alimentaire.

Le PVN, lui, est inhibiteur de la prise alimentaire - cette inhibition est levée par l'administration locale de noradrénaline et sa lésion bilatérale rend l'animal hyperphagique et obèse mais ne modifie pas la réponse alimentaire induite par le 2DG et l'insuline; il est donc exclu d'une réponse directe à la glucoprivation, mais pourrait avoir une action permissive sur l'activation des neurones du LH en fonction notam- ment des informations qu'il reçoit en provenance du rhombencéphale.

L'aphagie consécutive à une lésion bilatérale du LH a pu être attribuée à l'atteinte du système de fibres pallidofuges ou à celle des voies catécholaminergiques ascendantes parce que la lésion de ces voies en dehors du LH produit aussi une aphagie. De plus, une lésion des voies catécholaminergiques, et en particulier du faisceau nigrostrié, peut bloquer une réponse alimentaire induite par la glucoprivation. Ces données n'excluent cependant pas la possibilité d'une participation des neurones intrinsèques du $\mathrm{LH}$ à la régulation de la prise alimentaire.

Après quelques travaux qui mettaient en relation une activité neuronale LH spontanée et le comportement alimentaire, ceux de Katafuchi et al (1985) ont distingué des neurones phasiques activés chaque fois que l'animal introduit la tête dans la mangeoire et des neurones «toniques» activés pendant toute la durée du repas. L'activité de ces derniers semble donc liée au déclenchement et au maintien de «l'éveil alimentaire spécifique». En dehors du repas, ces neurones peuvent être activés par une glucoprivation centrale créée par injection de 2DG. Par ailleurs, des neurones qui répondent à la présentation de nourriture, mais seulement quand l'animal est susceptible de l'ingérer - autrement dit quand il a faim (Rolls et al, 1976; Burton et al, 1976; Kendrick et Baldwin, 1986) ont été mis en évidence chez le singe et le mouton.

Quelques études avaient mis en évidence dans le LH l'existence de neurones activés après injection périphérique de glucose mais les hyperglycémies ainsi induites étaient supraphysiologiques. Des expériences récentes (Himmi et al, 1988) ont montré qu'un tiers des neurones du $\mathrm{LH}$ répond soit à une légère hyper (en 
moyenne $36 \%$ ) soit à une légère hypoglycémie (en moyenne 10\%) induites, soit encore à des fluctuations spontanées de glycémie $(5-27 \%)$, et ce, avec une latence moyenne de $6 \mathrm{~min}$. Les auteurs ont montré que certains neurones répondent seulement aux variations périphériques de glycémie, d'autres seulement à l'application locale et d'autres enfin (1/3) aux 2 manipulations.

Ainsi, parmi les neurones "sensibles à la glycémie" certains peuvent être activés directement et d'autres indirectement. Les auteurs suggèrent que la réponse de ces derniers aux variations de glycémie pourrait être expliquée par la stimulation des afférences adrénergiques venant du rhombencéphale (NTS et AP).

Ainsi, au niveau du LH, lieu de convergence entre des afférences sensorielles externes et internes, il existe des neurones sensibles à des variations de glycémie d'une amplitude équivalente à celle du phénomène préprandial. Pour certains d'entre eux, l'activation est liée à leur glucosensibilité propre, pour d'autres elle relève d'un message transmis par d'autres sites directement sensibles à la glycémie. La prochaine étape de la démonstration, qui sera alors définitivement concluante, sera l'enregistrement simultané sur l'animal éveillé, de la prise alimentaire, de la glycémie et de cette activité neuronale.

\section{LE MÉCANISME LIPOSTATIQUE CHEZ LE SUJET HUMAIN OU ANIMAL}

Quand cesse le traitement (manœuvre expérimentale ou régime imposé) qui, chez un sujet animal ou humain, a induit progressivement un sur- ou un sous-poids, apparaît spontanément une hypo- ou une hyperphagie qui, d'abord intense, diminue peu à peu jusqu'au retour à l'état pondéral initial. La prise alimentaire est-elle le seul effecteur de cette régulation ? Certes non, l'adaptation du métabolisme basal, des dépenses thermogéniques et de l'activité physique à la surconsommation ou à la restriction est maintenant bien connue mais ne rend compte que d'une faible part du phénomène.

Un surpoids est un gain de tissu adipeux mais aussi de tissu maigre; de même, l'amaigrissement touche de pair bien que de façon très inégale - les 2 compartiments. Alors, qu'est-ce qui est régulé ? La masse maigre - et la masse grasse est reconstituée ce faisant - ou la masse grasse ? Peu de données permettent de répondre à cette question. Cependant, après un amaigrissement imposé, il semble d'une part que la masse grasse soit reconstituée d'abord (Harris et al, 1986), d'autre part qu'un appétit protéique spécifique ne se manifeste pas.

\section{Quel signal lie adiposité et prise alimentaire?}

Le signal qui agit sur la commande de prise alimentaire en fonction du niveau d'adiposité vient-il du tissu adipeux où estil seulement corrélé à l'adiposité ?

Dans le premier cas, l'information concerne-t-elle la masse des réserves ou la taille des adipocytes?

Plusieurs auteurs ont tenté de répondre à ces questions par l'ablation chirurgicale de dépôts adipeux chez l'animal. En général, ils constatent qu'une hypertrophie des dépôts restants compense la masse perdue (Liebelt et al, 1965; Schemmel et al, 1971; Chlouverakis et Hojuicki, 1974; Dark et al, 1984, 1985). Quand, après lipectomie, la prise alimentaire est mesurée, elle n'est pas trouvée augmentée (Schemmel et al, 1971; Dark et al, 1985). Deux auteurs (Bailey et Anderson, 1980) font cependant remarquer que, après lipectomie, 
les dépenses énergétiques sont ipso facto diminuées et qu'avec une prise alimentaire inchangée les animaux sont, en fait, transitoirement hyperphagiques. Néanmoins, il semble qu'à perte de poids égale, une lipectomie, donc une perte de masse, soit moins efficace dans l'induction d'une hyperphagie compensatrice qu'un amaigrissement général, donc une diminution de la taille des adipocytes.

\section{Si le signal émane bien du tissu adipeux, est-II nerveux ou humoral ?}

Le tissu adipeux a une innervation afférente (Fishman et Dark, 1987). II n'est pas possible de déterminer si ces fibres sensitives viennent des adipocytes ou du lit vasculaire, mais dans l'un ou l'autre cas, elles seraient susceptibles de véhiculer l'information. Néanmoins, les expériences de parabiose et de circulation croisée faites chez le rat sont en faveur d'un "facteur" circulant. Si l'un des partenaires parabiotiques est rendu hyperphagique et obèse, soit par lésion des noyaux ventromédians hypothalamiques, soit par stimulation électrique de l'hypothalamus latéral (Hervey, 1959; Parameswaran et al, 1977), l'autre est aphagique et perd du poids. La parabiose entre un rat Zucker obèse génétique ob/ob et l'hétérozygote non obèse rend aussi ce dernier aphagique et maigre (Harris et al, 1986). En revanche, le partenaire parabiotique d'un rat rendu obèse par gavage, s'il perd du poids, a cependant une prise alimentaire normale (Harris et Martin, 1984, 1986). Les auteurs eux-mêmes n'expliquent pas la différence. Peut-on évoquer le fait que le gavage constitue un court-circuit buccal? Peut-on penser qu'une obésité génétique ou une obésité hypothalamique sont des syndromes bien différents et plus complexes que celui qu'entraîne une simple surconsommation?
Le problème des parabioses n'est pas simple : on estime que les échanges plasmatiques sont tels qu'un échange total est effectué environ 10 fois en $24 \mathrm{~h}$. Tout facteur circulant est donc peu ou prou transféré d'un animal à l'autre et ce, en fonction de sa concentration et de sa demi-vie. L'analyse sérique de ces animaux a permis d'éliminer le rôle possible de la corticostérone, de l'insuline, de l'hormone de croissance, de la thyroxine, de la triodothyronine-reverse, du glucose, des acides gras libres ou enfin celui des corps cétoniques. Parmi les éléments dosés, seul le taux de triodothyronine a été trouvé élevé chez le rat gavé et son partenaire. Ce taux élevé de $T_{3}$ peut-il contribuer à la perte de masse grasse chez le partenaire non gavé (Harris et Martin, 1986) ?

Par ailleurs, Harris et Martin (1986) signalent 2 points particulièrement importants : la masse grasse du partenaire non gavé n'est pas atteinte avant le $23^{e} \mathrm{j}$ de gavage et le devient gravement ensuite, mais la masse maigre n'est jamais touchée. Le phénomène de fonte adipeuse semble donc bien dû à l'adiposité développée et non au processus de gavage.

La technique de circulation croisée, qui cependant présente l'inconvénient de n'être réalisable que sur un court laps de temps, permet un échange total du volume sanguin en $30 \mathrm{~min}$. Les résultats sont intéressants : un rat privé d'aliment pendant $5 \mathrm{~h}$, mis en circulation croisée avec un rat obèse, mange moins que s'il est mis en circulation avec un rat de poids normal; mais il mange beaucoup plus si son compère est amaigri (King, 1976). En résumé, il semble bien qu'un "facteur circulant" lié au niveau d'adiposité module la prise alimentaire. Mais il est bien sûr possible que ce "facteur non identifié" soit la conjonction de plusieurs facteurs agissant en synergie. 


\section{Niveau d'adiposité et tableau métabolique}

L'hypothèse d'un contrôle de la prise alimentaire par les carburants circulants proposée par Kennedy dès 1953 n'est pas démentie par les résultats acquis en quelque 40 ans (revues Scharrer et Langhans, 1990; Forbes, 1988). À la cessation du traitement ou du régime qui a induit chez un sujet, un niveau élevé d'adiposité, les taux plasmatiques d'acides gras libres, glycérol, corps cétoniques sont élevés. II a été montré pour tous ces métabolites qu'ils peuvent isolément ou en conjonction, inhiber la prise alimentaire via des récepteurs périphériques (hépatiques, particulièrement) dont l'information est transmise au SNC. II a aussi été montré que tous peuvent servir de carburants cellulaires, couvrir les dépenses courantes des tissus périphériques et, grâce en particulier à une épargne conséquente du glucose - cycle de Randle - (Randle et al, 1963), permettre aussi la couverture des dépenses du SNC : il y a mobilisation des réserves donc utilisation de carburants endogènes et l'animal est aphagique (Forbes, 1988).

À la cessation de la restriction alimentaire, les métabolites circulants sont principalement drainés par le foie et le tissu adipeux qui reconstituent leurs réserves. $\mathrm{Ce}$ retrait privilégié et la couverture nécessaire des dépenses courantes concourent à un épuisement rapide des métabolites absorbés lors d'un repas et ainsi, au déclenchement rapide du repas suivant : l'animal est hyperphagique.

Ainsi, il est possible d'envisager la modulation de la prise alimentaire comme la conséquence de la mobilisation des réserves pléthoriques ou de leur reconstitution quand leur niveau est bas. La question devient alors celle de la commande de ces mouvements de carburants, autrement dit, de la commande de la lipolyse ou de la li- pogenèse en fonction du niveau d'adiposité.

\section{Le témoin hypothalamique de l'état des réserves}

II y a 20 ans, Panksepp $(1972,1974)$ montrait qu'une charge intragastrique de glucose marqué avec un isotope radioactif $\left({ }^{14} \mathrm{C}\right)$ induisait au niveau de l'hypothalamus ventro-médian (VMH) un marquage durable de la fraction lipidique. Nicolaïdis et al (1974) suggéraient alors l'existence d'un modèle central de l'adiposité périphérique. Plus tard, Nicolaïdis (1980) observait que le contenu lipidique de l'hypothalamus variait comme celui des réserves périphériques. Récemment, une équipe de l'université de Géorgie (Kasser et al, 1985a, b; 1989a, b) s'est attaché à l'étude de la captation in vivo et à celle du métabolisme in vitro du glucose et des acides gras par l'hypothalamus latéral (LH) et l'hypothalamus ventro-médian $(\mathrm{VMH})$, et ce, en fonction de l'état des réserves et de l'état de faim de l'animal. Ces auteurs se sont intéressé à ces 2 zones tout particulièrement parce qu'un faisceau impressionnant de données recueillies en 50 ans de travaux avait montré leurs rôles dans la régulation du bilan d'énergie (revue Le Magnen, 1984). Le LH semble spécialement concerné par la commande de la prise alimentaire et le VMH par la gestion des réserves.

Kasser et al (1986) montrent que pour l'ensemble des zones testées du système nerveux central (SNC), sauf dans la région préoptique, la captation du glucose est diminuée (de $30 \%$ au niveau hypothalamique) et la captation du palmitate est augmentée (de $300 \%$ au niveau hypothalamique) par un jeûne de $24 \mathrm{~h}$.

Utilisant des rats gavés pendant quelques jours (10-20) à 50, 100 ou $150 \%$ de leurs prises spontanées et laissés ensuite 
en libre consommation, ces auteurs rapportent que :

- au niveau du LH (mais pas ailleurs dans le SNC) comme dans le foie, le taux du glucose oxydé par la voie du shunt du GABA est élevé quand le rat est surnourri ou qu'il surconsomme, diminué quand le rat est sousnourri ou qu'il sousconsomme, et $c e$, quel que soit l'état des réserves. Ainsi, au niveau du $\mathrm{LH}$, le taux de glucose oxydé par la voie du shunt du GABA semble refléter la prise alimentaire (le GABA est un neuromodulateur inhibiteur au niveau du $L H$ );

- au niveau du LH comme dans le foie, le taux d'oxydation des acides gras est bas quand le rat est obèse et s'élève peu à peu quand, à la cessation du surgavage, il reprend son poids; ce taux d'oxydation des acides gras ( $A G$ ) est élevé quand le rat est maigre et diminue quand, à la cessation du sous-gavage, l'animal reprend peu à peu son poids. Ainsi, au niveau du LH, le taux de palmitate oxydé semble dépendant du niveau des réserves;

- au niveau du VMH et comme dans le foie, le taux d'oxydation du glucose est bas quand le rat est gavé à $50 \%$ de sa ration spontanée;

- au niveau du VMH et aussi dans le foie, le taux d'oxydation du glucose par la voie des pentoses est élevé quand le rat est surnourri par gavage, qu'il soit seul ou en parabiose. La voie métabolique privilégiée est celle des pentoses; elle conduit à la synthèse d'acides gras. Les produits associés à cette voie des pentoses (par exemple NADPH) pourraient accroître l'activité électrique du VMH (Shimazu, 1981) et induire alors la mobilisation des réserves adipeuses via les efférences sympathiques décrites mais aussi via l'innervation sympathique du pancréas et des surrénales.

Sachant que l'activité de la voie des pentoses (au moins dans les tissus péri- phériques) est dépendante de l'état insulinique (Martin et Baldwin, 1971), et sachant aussi que le taux d'insuline (Baskin et al, 1983) et le nombre d'insulinorécepteurs sont élevés (Van Houten et al, 1979) au niveau du $\mathrm{VMH}$, il est possible de suspecter le rôle du niveau d'insulinémie dans ce phénomène (voir ci-dessous).

II est tout à fait intéressant de remarquer que l'activité de la voie des pentoses est trouvée élevée de façon égale chez le rat surnourri par gavage et son partenaire parabiotique (Kasser et al, 1989a). Si l'activité électrique du VMH est fonction de l'activité de cette voie métabolique, il n'est pas étonnant que ce partenaire soit maigre. Le mécanisme lipolytique agissant chez ce partenaire ne serait pas un facteur circulant émanant du tissu pléthorique du rat surnourri par gavage mais serait lié à l'activité accrue de son propre VMH. Cet animal, rappelons-le, mange normalement et est couplé à un animal gavé à $200 \%$. II serait tout à fait intéressant de suivre l'évolution des tableaux métaboliques de l'un et l'autre des rats parabiotiques au cours du temps et en fonction des séances de gavage.

En 1984, Le Magnen écrivait : «tout se passe comme si l'activation du VMH, parallèle à ou déterminée par une replétion limite de la réserve lipidique, commandait par les voies sympathiques descendantes une lipolyse correctrice...". À la lumière des travaux de l'équipe de Kasser, il semble que l'activation ou l'inhibition (quand il y a déplétion de la masse adipeuse) soient entraînées par le métabolisme propre de l'hypothalamus ventromédian qui synthétise des acides gras libres comme le foie et les stocke comme le tissu adipeux.

\section{Rôle de l'insuline}

Dans toutes les espèces et toutes les situations où la recherche a été effectuée, le 
taux d'insuline plasmatique est corrélé à l'adiposité (revue Woods et Porte, 1978). De plus, bien que fort atténuées, les variations de l'insulinémie sont transmises au liquide céphalorachidien (LCR) avec un certain délai. II se pourrait donc que l'insuline soit le messager informant le SNC de l'état des réserves. II existe des récepteurs à l'insuline dans le SNC, en particulier dans les sites périventriculaires comprenant l'hypothalamus et l'area postrema (revue Posner, 1987). Des récepteurs insuliniques sont présents dans les capillaires cérébraux et pourraient jouer le rôle de transporteurs d'insuline par transcytose (Posner, 1987; Woods, 1988) permettant alors un transport rapide (Woods, 1988).

Les premiers, Woods et al (1979) montraient qu'une perfusion d'insuline intracérébroventriculaire (ICV) chez le singe, provoquait hypophagie et perte de poids. Depuis, beaucoup de travaux réalisés en particulier chez le rat, avec des doses quelquefois extraphysiologiques perfusées dans les ventricules ou administrées au niveau hypothalamique, ont donné des résultats contradictoires (revue Scharrer et Langhans, 1990). Une récente étude, convaincante, porte sur l'effet de perfusions d'insuline à doses physiologiques dans divers sites hypothalamiques ( $\mathrm{Mc}$ Gowan et al, 1990). Elle montre que les perfusions dans le VMH provoquent une diminution de la prise alimentaire et du poids corporel dépendant de la dose d'insuline perfusée. Les mêmes perfusions faites dans la région périfornicale médiane sont moins efficaces, faites dans le LH pas du tout. Par ailleurs, il est intéressant de remarquer que les perfusions d'insuline ICV efficaces chez l'animal ayant un régime équilibré ne le sont pas chez l'animal maintenu sur un régime hyperlipidique (Arase et al, 1988) ou chez l'animal à jeun (Plata-Salaman et Oomura, 1986). Chez ce dernier, par ailleurs, on a montré une di- minution de la liaison insulinique au niveau du VMH. Ceci permet d'étayer l'hypothèse du rôle de l'insuline dans le métabolisme glucidique du VMH et donc, via l'activité de l'oxydation du glucose par la voie des pentoses, le rôle de l'insuline dans la commande lipolytique correctrice de l'adiposité.

\section{CONCLUSION}

L'acte alimentaire stimulé par un signal glucopénique est la recharge périodique d'un petit réservoir d'énergie externe à l'organisme (tube digestif). Peu à peu absorbés, les métabolites ingérés vont entrer dans le pool des carburants circulants qui assurent la couverture des dépenses courantes. II existe des arguments pour penser que le métabolisme propre des neurones du centre hypothalamique de gestion des réserves internes (hépatique, adipeuse) conditionne leur activité. II semble, après les travaux de l'équipe de Kasser, que cette activité puisse refléter l'état métabolique périphérique (en particulier le métabolisme hépatique) et l'état des réserves. Cette activité commanderait alors de façon adaptée, soit le retrait de carburants vers les réserves à partir du pool circulant, soit l'apport de carburants à ce pool à partir des réserves. Ces mouvements précipitent ou retardent la survenue du signal glucopénique et donc celle de la prise alimentaire suivante.

Ce retrait ou cette addition au pool circulant alternent fonctionnellement dans un cycle quotidien : il est bien établi que pendant la moitié du nycthémère où il est actif, le sujet animal ou humain ingère plus d'énergie qu'il n'en dépense et augmente ses réserves; celles-ci sont mobilisées pendant la période de repos au cours de laquelle le sujet prend peu ou pas de repas, mais assure cependant la couverture des dépenses basales. Chez l'animal 
de laboratoire en conditions stables, bilans positif et négatif s'équilibrent strictement à l'échelle du nycthémère. Chez le sujet humain, pour des raisons multiples, les unes bien cernées, les autres moins, l'équilibre strict intervient rarement au terme des 24 $h$, mais le plus souvent, au terme de quelques j.

\section{RÉFÉRENCES}

Adachi A, Kobashi M (1985) Chemosensitive neurons within the area postrema. Neurosci Lett 55, 137-140

Adachi A, Kobashi M (1988) Electrophysiological analysis of chemosensitive neurons within the area postrema of the rat. In: Transduction and cellular mechanisms in sensory receptors (Hamann, Iggo, ed), Progress in Brain Research, Elsevier Sci Publ BV Biomedical Division, Amsterdam, 77, 77-85

Adachi A, Shimizu N, Oomụra $Y$, Kobashi M (1984) Convergence of hepatoportal glucose-sensitive afferent signals to glucosesensitive units within the nucleus of the solitary tract. Neurosci Lett 46, 215-218

Arase K, Fisler JS, Shargill NS, York DA, Bray GA (1988) Intracerebroventricular infusions of 3-OHB and insulin in a rat model of dietary obesity. Am J Physiol 255, R974-981

Bailey JW, Anderson DB (1980) Rate of fat compensation and growth efficiency of lipectomized Sprague-Dawley rats. J Nutr 110, 1785-1792

Baskin DG, Porte D, Guest K, Dorsa DM (1983) Regional concentrations of insulin in the rat brain. Endocrinology 112, 898-903

Burton MJ, Rolls ET, Mora F (1976) Effects of hunger on the responses of neurons in the lateral hypothalamus to the sight and taste of food. Exp Neurol 51, 668-677

Campfield LA, Brandon SP, Smith FJ (1985) On line continuous measurement of blood glucose and meal pattern in free-feeding rats: the role of glucose in meal initiation. Brain Res Bull 14, 605-616

Campfield LA, Smith FJ (1986) Blood glucose and meal initiation : a role for insulin. Soc Neurosci Abstr 12,109
Campfield LA, Smith FJ, Brandon SP (1984) Partial blockade of pre-meal decline in blood glucose delays feeding. Neurosci Soc Meeting NY, Neural \& metabolic bases of feeding. 7-9 oct

Chlouverakis C, Hojuicki D (1974) Lipectomy in obese hyperglycemic mice (ob/ob). Metabolism 23, 133-137

Dark J, Forger NG, Zucker I (1984) Rapid recovery of body mass after surgical removal of adipose tissue in ground squirrel. Proc Natl Acad Sci USA 81, 2270-2277

Dark J, Forger NC, Stern JS, Zucker I (1985) Recovery of lipid mass after surgical removal of adipose in ground squirrel. Am J Physiol 249, R73-78

De Castro JM (1981) The stomach energy content governs meal patterning in the rat. Physiol Behav 26, 795-798

Even P, Nicolaïdis S (1984) Le métabolisme de fond : définition et dispositif de sa mesure. CR Séances Acad Sci (Paris) 298, 261-266

Even $P$, Coulaud H, Nicolaïdis S (1988) Integrated metabolic control of food intake after 2deoxy-D-glucose and nicotinic acid injection. Am J Physiol 255, R82-90

Fishman RB, Dark J (1987) Sensory innervation of white adipose tissue. Am J Physiol 253, R942-944

Forbes JM (1988) Metabolic aspects of the regulation of voluntary food intake and appetite. Nutr Res Rev 1, 145-168

Fuller RW, Diller ER (1970) Diurnal variation of liver glycogen and plasma free fatty acids in rats fed ad lib or single daily meal. Metabolism 19, 226-229

Geiselman PJ, Novin D (1982) The role of carbohydrates in appetite, hunger and obesity. Appetite 3, 203-223

Glick Z, Modan M (1977) Behavioural compensatory responses to continuous duodenal and upper ileal glucose infusion in rats. Physiol Behav 19, 703-705

Harris RBS, Martin RJ (1984) Specific depletion of body fat in parabiotic partners of tubefed obese rats. Am J Physiol 247, R380-386

Haris RBS, Martin RJ (1986) Metabolic response to a specific lipid-depleting factor in parabiotic rats. Am J Physiol 250, R276-286 
Harris RBS, Kasser TR, Martin RJ (1986) Dynamics of recovery of body composition after overfeeding, food restriction or starvation of mature female rats. J Nutr 116, 2536-2546

Hervey GR (1959) The effect of lesions in the hypothalamus in parabiotic rats. $J$ Physiol 145, 343-352

Himmi T, Boyer A, Orsini JC (1988) Changes in lateral hypothalamic neuronal activity accompanying hyper- and hypoglycemias. Physiol Behav 44, 347-355

Himmi T (1989) Sensibilité de l'hypothalamus latéral aux variations de glycémie. Rôle dans la régulation du comportement alimentaire. Thèse de Doctorat, Université de Provence, Marseille

Kasser TR, Deutch A, Martin RJ (1986) Uptake and utilization of metabolites in specific brain sites relative to feeding status. Physiol Behav 36, 1161-1165

Kasser TR, Harris RBS, Martin RJ (1985a) Level of satiety: fatty acid and glucose metabolism in three brain sites associated with feeding. Am J Physiol 248, R447-452

Kasser TR, Harris RB, Martin RJ (1985b) Level of satiety: GABA and pentose shunt activities in three brain sites associated with feeding. Am J Physiol 248, R453-458

Kasser TR, Harris RB, Martin RJ (1989a) Site ofaction of putative lipostatic factor: hypothalamic metabolism of parabiotic rats. $A m ~ J$ Physiol 257, R224-228

Kasser TR, Harris RB, Martin RJ (1989b) Level of satiety: in vitro energy metabolism in brain during hypophagic and hyperphagic body weight recovery. Am J Physiol 257, R13221327

Katafuchi T, Oomura Y, Yoshimatsu H (1985) Single neuron activity in the rat lateral hypothalamus during 2-deoxy-D-glucose induced and natural feeding behavior. Brain Res 359 , 1-10

Kendrick KM, Baldwin BA (1986) Characterization of neuronal responses in the zona incerta of the subthalamic region of the sheep during ingestion of food and liquid. Neurosci Lett 63, 237-243

Kennedy GG (1953) The role of depot fat in the hypothalamic control of food intake in the rat. Proc $R$ Soc B140, 578-592
King KR (1976) Lipostatic control of body weight: evidence of humoral mediation. Physiol Psychol 4, 405-408

Lagaillarde M (1985) Contribution à l'étude du stimulus métabolique initiateur du repas chez le rat. Thèse de Doctorat, Université Paris VI

Le Magnen J (1984) Bases neurobiologiques du comportement alimentaire. In: Neurobiologie des comportements (Delacour J, ed) Hermann, Paris, 1-54

Le Magnen J (1988) Faim et satiété. Prise alimentaire et régulation nutritionnelle. Encycl Méd Chir (Paris-France). Glandes-Nutrition, 1-14

Le Magnen J, Devos M (1980) Variations of meal-to-meal liver glycogen in rats. Neurosci Biobehav Rev 4, suppl 1, 29-32

Liebelt RA, Ichinoe S, Nicholson N (1965) Regulatory influences of adipose tissue on food intake and body weight. Ann NY Acad Sci 131, 559-582

Louis-Sylvestre J, Le Magnen J (1980a) A fall in blood glucose level precedes meal onset in free feeding rats. Neurosci Biobehav Rev (suppl 1), 13-15

Louis-Sylvestre J, Le Magnen J (1980b) Palatability and preabsorptive insulin release. Neurosci Biobehav Rev 4 (suppl 1), 43-46

Louis-Sylvestre J, Larue-Achagiotis C, Michel A, Houssin D (1990) Feeding pattern of livertransplanted rats. Physiol Behav 48, 321-326

Martin RJ, Baldwin RJ (1971) Effects of insulin and anti-insulin serum treatments on levels of metabolites in rat mammary glands. Endocrinology 88, 868-871

Mayer $\mathrm{J}$ (1955) Regulation of energy intake and the body weight: the glucostatic theory and the lipostatic hypothesis. Ann NY Acad Sci $73,15-42$

McGowan MK, Andrews KM, Kelly J, Grossman SP (1990) Effects of chronic intrahypothalamic infusion of insulin on food intake and diurnal meal patterning in the rat. Behav Nourosci 104, 2, 373-385

Mei N (1978) Vagal glucoreceptors in the small intestine of the cat. J Physiol (Lond) 282, 485-506

Mei N, Arlhac A, Boyer A (1981) Nervous regulation of insulin release by the intestinal vagal glucoreceptors. J Auton Nerv Syst 10a, 275278 
Mei N, Perrin J, Crousillat J, Boyer A (1984) Comparison between the properties of the vagal and splanchnic glucoreceptors of the small intestine. Involvement in insulin release. J Auton Nerv Syst 1984, 275-278

Nicolaïdis S (1974) Short term and long term regulation of energy balance. XXVI Int Cong Physiol Sci (New Delhi). In: Proc Int Union Physiol Sci 10, 122

Nicolaïdis S (1980) Hypothalamic convergence of external and internal stimulation leading to early ingestive and metabolic responses. Brain Res Bull 5, 97-101

Nicolaidis S, Petit M, Polonowski J (1974) Étude du rapport entre la régulation de la masse adipeuse corporelle et la composition lipidique de ses centres régulateurs. $C R$ Séances Acad Sci Paris 278, 1393-1396

Nijijima A (1969) Afferent impulse discharges from glucoreceptors in the liver of the guinea pig. Ann NY Acad Sci 157, 690-700

Panksepp J (1972) Hypothalamic radioactivity after intragastric glucose ${ }^{14} \mathrm{C}$ in rats. $A m \mathrm{~J}$ Physiol 223, 396-401

Panksepp J (1974) Hypothalamic regulation of energy balance and feeding behavior. Fed Proc 33, 1150-1165

Parameswaran SV, Steffens AB, Hervey GR, de Ruiter L (1977) Involvement of a humoral factor in regulation of body weight in parabiotic rats. Am J Physiol 232, R150-157

Perrin J, Crousillat J, Mei N (1981) Assessment of true splanchnic glucoreceptors in the jejunum-ileum of the cat. Brain Res Bull 7, 625628

Plata-Salaman OR, Oomura $Y$ (1986) Effect of intra-third ventricular administration of insulin on food intake after food deprivation. Physiol Behav 37, 735-739

Posner BI (1987) Insulin interaction with the central nervous system: nature and possible significance. Proc Nutr Soc 46, 97-103

Puls W, Keup U, Krause HP, Thomas G, Hoffmeister $F$ (1977) Glucosidase inhibition: a new approch to the treatment of diabetes, obesity and hyperlipoproteinemia. Naturwissenschaften 64, 536-537

Randle RJ, Garland PB, Hales CN, Newsholme EA (1963) The glucose fatty acid cycle. Its role in insulin sensitivity and the metabolic disturbances of diabetes mellitus. Lancet i, 785-789

Ritter RC, Slusser PG, Stone S (1981) Glucoreceptors controlling feeding and blood glucose: location in the hindbrain. Science 213, $451-453$

Rolls ET, Burton MJ, Mora F (1976) Hypothalamic neuronal responses associated with the sight of food. Brain Res 111, 53-66

Scharrer E, Langhans W (1990) Mechanisms for the effect of body fat on food intake. $\mathrm{In}$ : The control of body fat content (Forbes, Hervey, eds) Smith Gordon, 63-86

Schemmel R, Mickelsen O, Pierce SA, Johnson JT, Schirmer RG (1971) Fat depol removal, food intake, body fat, and fat depot weights in obese rats. Proc Soc Exp Biol Med 136, 1269-1273

Shimazu T (1981) Central nervous system regulation of liver and adipose metabolism. Diabetologia 20, 343-356

Steffens $A B$ (1969) The influence of insulin injections and infusions on eating and blood glucose level in the rat. Physiol Behav 4, 823-828

Steffens AB (1970) Plasma insulin content in relation to blood glucose level and meal pattern in the normal and hypothalamic hyperphagic rat. Physiol Behav 5, 147-151

Strubbe JH, Bouman PR (1978) Plasma insulin patterns in the unaesthetized rat during intracardial infusion and spontaneous ingestion of graded loads of glucose. Metabolism 27, 341-351

Strubbe JH, Stefiens AB (1977) Blood glucose levels in portal and peripheral circulation and their relation to food intake in the rat. Physiol Behav 19, 303-307

Strubbe JH, Steffens AB, De Ruiter IJ (1977) Plasma insulin and the time pattern of feeding in the rat. Physiol Behav 18, 81-86

Thomas DW, Mayer J (1978) Meal size as a determinant of food intake in normal and hypothalamic obese rats. Physiol Behav 21, 113118

Van Houten M, Posner BI, Korpiwa BM, Brawer JR (1979) Insulin-binding sites in rat brain: in vivo localization to circumventricular organs by quantitative radioautography. Endocrinology $105,666-673$ 
Van Itallie TB, Beaudoin R, Mayer J (1953) Arteriovenous glucose differences, metabolic hypoglycemia and food intake in man. $J$ Clin Nutr 1, 208-216

Weingarten HP (1983) Conditioned cues elicit feeding in sated rats: a role for learning in meal initiation. Science 220, 431-433

Woods SC (1988) New perspectives on the role of insulin as an adiposity signal to the brain, VI-4A. In: Third Benjamin Franklin Lafayette
Seminar (Epstein, Nicolaïdis, Stellar, eds) La Napoule

Woods SC, Porte DJ (1978) The central nervous system, pancreatic hormones, feeding, and obesity. Adv Metab Disord 9, 283-312

Woods SC, Lotter EE, McKay LD, Porte D Jr (1979) Chronic intercerebroventricular infusion of insulin reduces food intake and body weight of baboons. Nature (Lond) 282, 503505 